\title{
Could triglyceride to high density lipoprotein-cholesterol ratio predict hepatosteatosis?
}

\author{
Ozge Kurtkulagi $^{1}$, Satilmis Bilgin ${ }^{1}$, Gizem Bakir Kahveci $^{1}, \quad$ Burcin Meryem Atak Tel ${ }^{1}$, \\ Mehmet Ali Kosekli \\ ${ }^{1}$ Department of Internal Medicine, Bolu Abant Izzet Baysal University, School of Medicine, Bolu, Turkey \\ ${ }^{2}$ Department of Internal Medicine and Gastroenterology, Bolu Abant Izzet Baysal University, School of Medicine, Bolu, \\ Turkey
}

\section{ABSTRACT}

\begin{abstract}
Aim: The triglyceride / HDL cholesterol (TG/HDL-c) ratio is increased in a variety of diseases including, coronary heart disease and type 2 diabetes mellitus. However, its role in non-alcoholic hepatosteatosis is not well understood. In present study, we aimed to compare the TG/HDL-c levels of the patients with nonalcoholic hepatosteatosis to those of the healthy subjects.

Methods: Medical data of the patients with non-alcoholic hepatosteatosis whom presented to the outpatient internal medicine clinics of our institution were retrospectively analyzed. Healthy subjects whom admissions to our clinics were due to check up were enrolled to the study as control group. TG/HDL-c of the groups compared.
\end{abstract}

Results: TG/HDL-c level of the liver steatosis group (5 (2-22) \%) was higher than the control group (2.7 (1$8) \%),(\mathrm{p}<0.001)$. TG/HDL-c was significantly and positively correlated with fasting blood glucose ( $\mathrm{r}=0.31$, $\mathrm{p}<0.001), \mathrm{C}$ - reactive protein $(\mathrm{r}=0.25, \mathrm{p}<0.001)$ and LDL-cholesterol $(\mathrm{r}=0.3, \mathrm{p}<0.001)$ levels. A TG/HDL-c value greater than $3.1 \%$ has $91 \%$ sensitivity and $77 \%$ specificity in detecting hepatosteatosis.

Conclusions: We suggest that TG/HDL-c ratio could be a useful marker of non-alcoholic hepatosteatosis due to its inexpensive and easy to assess nature.

Key words: Liver steatosis, hepatosteatosis, inflammation, triglyceride, HDL cholesterol.

$\triangle D r$. Ozge Kurtkulagi

Department of Internal Medicine, Bolu Abant Izzet Baysal University, School of Medicine, Bolu, Turkey

E-mail: ozgekurtkulagi@gmail.com

Received: 2021-01-14 / Revisions: 2021-01-30

Accepted: 2021-02-09 / Published online: 2021-07-01

\section{Introduction}

Liver steatosis, as known as hepatosteatosis, is defined as abnormal lipid accumulation in liver cells. The pathogenesis of the hepatosteatosis includes inflammation, metabolic disorder and hyperlipidemia. Possible contributing factors to non-alcoholic hepatosteatosis are metabolic syndrome, dyslipidemia, obesity, type 2 diabetes mellitus and insulin resistance.

Recent researches studied numerous inflammatory and metabolic indices in patients with non-alcoholic hepatosteatosis. These include; $\mathrm{C}$ - reactive protein, mean platelet volume, platelet distribution width, mean platelet volume to platelet count ratio and neutrophil/lymphocyte ratio [1-5]. Novel studies pointed out the relationship between triglyceride/ HDL cholesterol (TG/HDL-c) ratio in various conditions. Elevated TG/HDLc level was considered as a risk factor for coronary heart disease in male population [6]. 
In a study from Japan, authors introduced TG/HDL-c ratio as a useful tool in predicting small LDL particles [7]. Moreover, it has been suggested that increased TG/HDL-c levels in young adults may serve as a predictor of insulin resistance and higher cardiometabolic risk [8].

In present study, we aimed to compare the TG/HDL-c levels of the patients with nonalcoholic hepatosteatosis to those of the healthy subjects.

\section{Materials and methods}

After receiving ethical approval from the local ethics committee (Date and decision no: 2020/311), medical data of the patients with non-alcoholic hepatosteatosis whom presented to the outpatient internal medicine clinics of our institution between October 2018 and October 2020 were retrospectively analyzed. Healthy subjects whom admissions to our clinics were due to check up within the same time period were enrolled to the study as control group. Exclusion criteria were as follows: hepatitis, alcohol consumption, active inflammatory diseases, hyperlipidemic syndromes, recent infection and those with a history of lipid lowering drug use. Hepatosteatosis was determined by ultrasound scan.

Age, gender, body weight, height, fasting blood glucose (FBG), serum creatinine, LDL cholesterol, HDL cholesterol, triglyceride, aspartate and alanine transaminases (AST and ALT) and c-reactive protein (CRP) levels were obtained and recorded from the institutional database and patients' files. Biochemical analyses were held with Architect c8000 analyzer (Abbott Inc. Lake Forest, IL, USA). White blood cell count (WBC), hemoglobin $(\mathrm{Hb})$, hematocrit $(\mathrm{Htc})$ and platelet count (plt) were also recorded. Automatic analyzer of $\mathrm{LH}$ 780 model of Beckman Coulter device (Beckman Coulter Inc.; Bre CA) was used for complete blood count analyses. A TG/HDL-c level was obtained by the ratio of triglyceride/HDL-cholesterol. Body mass index is calculated by the division of weight by the square of height. Data of the patients with liver steatosis and the control subjects were compared.

\section{Statistical analysis}

Data were analyzed with SPSS software (SPSS 15.0; SPSS Inc., Chicago, IL, USA). Kolmogorov-Smirnov test was used in observing the distribution (normal or not) of the variables in study and control groups in terms of normal analyzed with. Variables with normal distribution were compared with independent samples $t$ test and expressed as mean \pm standard deviation (SD). Mann- Whitney U test is used in comparison of the variables without normal distribution and these variables were expressed as median (min.-max.). Chi-square test is used in comparison of categorical variables between study and control subjects. Correlation between studies variables were conducted with Pearson's correlation analysis. Receiver Operative Characteristics (ROC) analyze used in determination of cut-off values of study variables in predicting NAFLD. Statistical significance was set when the $\mathrm{p}$ value was lower than 0.05 .

\section{Results}

A total of 496 subjects, 267 in liver steatosis and 229 in control group, were enrolled to the study. Mean ages of the liver steatosis and control groups were $45.5 \pm 12$ years and $44 \pm$ 10 years, respectively $(p=0.09) .131 \quad(49 \%)$ were women and $136(51 \%)$ were men in the liver steatosis group whereas 96 (42\%) were women and 133 (58\%) were men in control group. Gender of the liver steatosis and control groups were not statistically different $(p=0.11)$. 


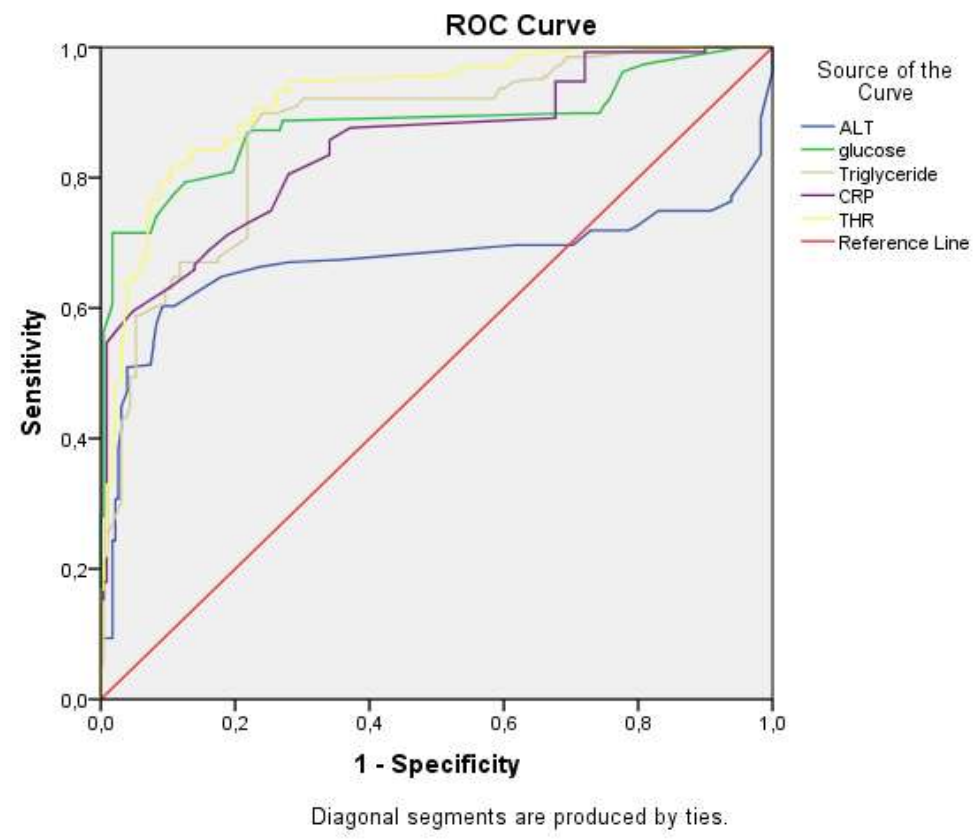

Figure 1. ROC curves of ALT, fasting glucose, triglyceride, CRP, and THR in determination of liver steatosis
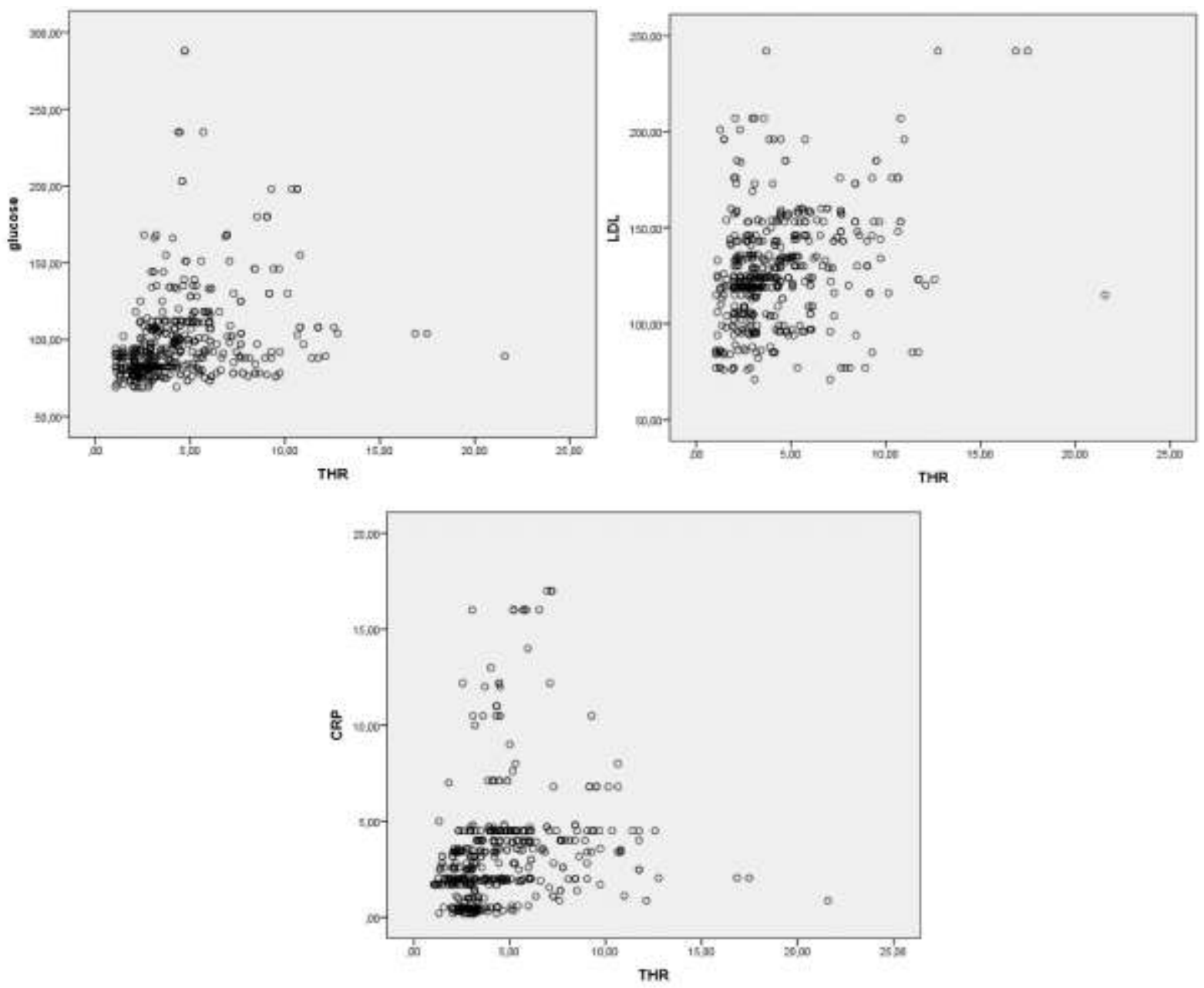

Figure 2. Correlations of THR with fasting glucose, LDL- cholesterol and CRP level. 
The WBC $(p=0.11), \mathrm{Hb} \quad(p=0.26), \quad \mathrm{Htc}$ $(p=0.08)$, PLT $(p=0.19)$, serum creatinine $(p=0.1)$ and AST $(p=0.54)$ levels of the liver steatosis and control groups were not statistically different.

The ALT $(p<0.001)$, FPG $(p<0.001)$, triglyceride $\quad(p<0.001), \quad$ LDL-cholesterol $(p<0.001)$ and CRP $(p<0.001)$ levels were significantly increased and HDL-cholesterol $(p<0.001)$ was significantly decreased in liver steatosis group compared to the control subjects. TG/HDL-c level of the liver steatosis group [5 (2-22) \%] was higher than the TG/HDL-c level of the control group [2.7 (1-8) $\%]$, and this difference was statistically significant $(p<0.001)$.

In Roc analysis test, a TG/HDL-c value greater than $3.1 \%$ have $91 \%$ sensitivity and $77 \%$ specificity in detecting hepatosteatosis, which is better than other variables, i.e., ALT, FPG, triglyceride and CRP (Figure 1).

In Pearson's correlation analysis test, TG/HDLc was significantly and positively correlated with FPG $(r=0.31, p<0.001), C R P(r=0.25$, $p<0.001)$ and LDL-cholesterol $(r=0.3$, $p<0.001$ ) levels (Figure 2).

\section{Discussion}

Main finding of present retrospective study is that $\mathrm{TG} / \mathrm{HDL}-\mathrm{c}$ ratio could be a promising indicator of liver steatosis in general population. Since it has significant correlation with inflammatory (CRP) and metabolic (FPG and LDL-cholesterol) markers of hepatosteatosis, and has stunning sensitivity and specificity in determination of the disease, it may have substantial utility in clinical practice.

Recent studies in literature focused on the usefulness of TG/HDL-c ratio in various clinical conditions. Authors suggested the TG/HDL-c ratio as a novel prognostic marker in patients receiving peritoneal dialysis treatment since elevated THR was correlated with worse survival in this population [9]. Chronic renal insufficiency, like liver steatosis, is a condition characterized with increased inflammatory burden. Therefore, increased levels of TG/HDL-c were noted in patients with liver steatosis compared to controls in present work.

The role of TG/HDL-c ratio has been studied in gestational diabetes mellitus, too. A Chinese study reported that elevated first trimester TG/HDL-c levels could predict the development of gestational diabetes mellitus [10]. Both pregnancy, and gestational diabetes mellitus are characterized with insulin resistance and increased inflammation. Liver steatosis is also associated with insulin resistance and increased inflammatory burden. Therefore, elevated TG/HDL-c levels which reported in our study is a compatible finding with current medical literature.

Increased level of TG/HDL-c has been found to be a risk factor for inflammatory breast cancer in Schairer et al's study, which reported a 1.7 odds ratio for the THR level greater than $2.5 \%$ [11]. Cancer is also promoted by inflammatory reactions and cause chronic, low grade, continuous inflammatory load. Therefore, as an inflammatory condition, liver steatosis caused an elevation in TG/HDL-c levels in our study. The TG/HDL-c ratio has been proposed as a prognostic tool in coronary artery disease $[6,12]$.

Moreover, high TG/HDL-c levels were associated with abnormal heart rate recovery, which is a marker of decreased parasympathetic activity and a predictor of allcause mortality [13].

High TG/HDL-c ratio is associated with deteriorated insulin sensitivity [14]. Elevated TG/HDL-c is suggested to be a predictor of incident type 2 diabetes mellitus in male 
subjects [15]. A similar association between diabetes mellitus and increased TG/HDL-c has been reported in obese postmenopausal women [16]. Since liver steatosis is associated with insulin resistance and inflammation, as diabetes mellitus do, increased levels of TG/HDL-c in patients with liver steatosis is not a coincidental finding.

Retrospective design and moderately large study population are two possible limitations of our work. However, to the best of our knowledge, this is the first study reported high sensitivity and specificity of TG/HDL-c ratio in detecting liver steatosis.

In conclusion, we suggest that TG/HDL-c ratio could be a useful marker of liver steatosis due to its inexpensive and easy to assess nature.

Funding: The author(s) received no financial support for the research, authorship, and/or publication of this article.

Conflict of Interest: The authors declare that they have no conflict of interest.

Ethical statement: The study was approved by the Local Ethics Committee of University (Date and decision no: 2020/311), and written informed consent was obtained from each subject.

\section{Open Access Statement}

This is an open access journal which means that all content is freely available without charge to the user or his/her institution under the terms of the Creative Commons Attribution NonCommercial License (http://creativecommons.org/licenses/bync/4.0). Users are allowed to read, download, copy, distribute, print, search, or link to the full texts of the articles, without asking prior permission from the publisher or the author.

\section{References}

[1]Keenan T, Blaha MJ, Nasir K et al. Relation of uric acid to serum levels of highsensitivity C-reactive protein, triglycerides, and high-density lipoprotein cholesterol and to hepatic steatosis. Am J Cardiol. 2012; 110 (12): 1787-92.

[2]Aktas G, Alcelik A, Tekce BK et al. Mean platelet volume and red cell distribution width in hepatosteatosis. Natl J Med Res. 2013; 3 (3): 264-66.

[3]Aktas G, Duman TT, Kurtkulagi O et al. Liver Steatosis is Associated Both with Platelet Distribution Width, Neutrophil/Lymphocyte and Monocyte/Lymphocyte Ratios. Prim Health Care Open Access. 2020; 10 (4): 1-4.

[4]Kosekli MA, Erkus E, Kocak MZ. Mean Platelet Volume to Platelet ratio as a promising marker of hepatosteatosis. Exp Biomed Res. 2018; 1 (2): 55-59.

[5]Rodak AP, Kiciak S, Tomasiewicz K. Neutrophil-lymphocyte ratio and mean platelet volume as predictive factors for liver fibrosis and steatosis in patients with chronic hepatitis B. Ann Agricul Environ Med. 2018; 25 (4): 690-92.

[6]Hadaegh F, Khalili D, Ghasemi A et al. Triglyceride/HDL-cholesterol ratio is an independent predictor for coronary heart disease in a population of Iranian men. Nutr Metab Cardiovasc Dis. 2009; 19 (6): 401-08. [7]Maruyama C, Imamura $\mathrm{K}$, Teramoto $\mathrm{T}$. Assessment of LDL particle size by triglyceride/HDL-cholesterol ratio in nondiabetic, healthy subjects without prominent hyperlipidemia. J Atheroscleros Thrombos. 2003; 10 (3): 186-91.

[8]Murguía-Romero M, Jiménez-Flores JR, Sigrist-Flores SC et al. Plasma triglyceride/HDL-cholesterol ratio, insulin resistance, and cardiometabolic risk in 
young adults. J Lipid Res. 2013; 54 (10): 2795-99.

[9]Xia W, Yao X, Chen $Y$ et al. Elevated TG/HDL-C and non-HDL-C/HDL-C ratios predict mortality in peritoneal dialysis patients. BMC Nephrol. 2020; 21 (1): 324.

[10]Liu PJ, Liu Y, Ma L et al. The Predictive Ability of Two Triglyceride-Associated Indices for Gestational Diabetes Mellitus and Large for Gestational Age Infant Among Chinese Pregnancies: A Preliminary Cohort Study. Diab Metab Syndr Obes: targets and therapy 2020; 13: 2025-35.

[11] Schairer C, Laurent CA, Moy LM et al. Obesity and related conditions and risk of inflammatory breast cancer: a nested casecontrol study. Breast Cancer Res Treat. 2020; 183 (2): 467-78.

[12] da Luz PL, Favarato D, Faria-Neto JR, Jr. et al. High ratio of triglycerides to HDLcholesterol predicts extensive coronary disease. Clinics (Sao Paulo, Brazil) 2008; 63 (4): 427-32.

[13] Shishehbor MH, Hoogwerf BJ, Lauer MS. Association of triglyceride-to-HDL cholesterol ratio with heart rate recovery. Diabetes Care 2004; 27 (4): 936-41.

[14]Gonzalez-Chavez A, Simental-Mendia LE, Elizondo-Argueta S. Elevated triglyceride/HDL-cholesterol ratio associated with insulin resistance. Cirugia y cirujanos. 2011; 79 (2): 126-31.

[15] Vega GL, Barlow CE, Grundy SM et al. Triglyceride-to-high-density-lipoproteincholesterol ratio is an index of heart disease mortality and of incidence of type 2 diabetes mellitus in men. J Invest Med. 2014; 62 (2): 345-9.

[16] Karelis AD, Pasternyk SM, Messier L et al. Relationship between insulin sensitivity and the triglyceride-HDL-C ratio in overweight and obese postmenopausal women: a
MONET study. Appl Physiol Nutr Metab. 2007; 32 (6): 1089-96. 